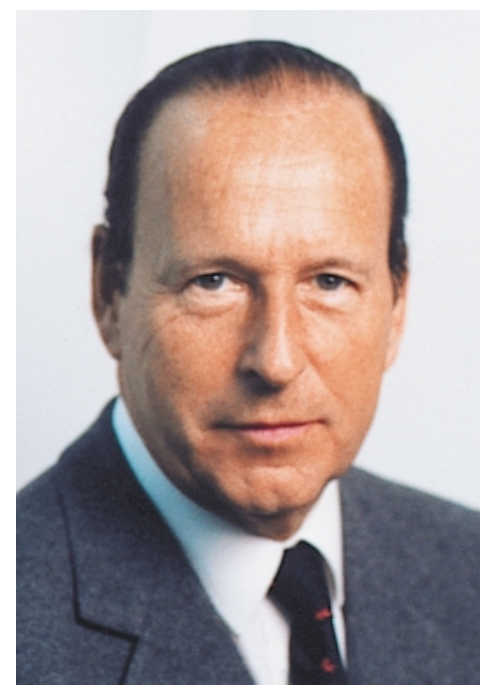

\title{
Les accords bilatéraux: projet mal conçu, pour les médecins également
}

\author{
J. H. Dunant \\ Correspondance: \\ Dr Jean Henri Dunant, p.-d. \\ Conseiller national UDC \\ Luftmattstrasse 12 \\ CH-4052 Bâle
}

En principe, les accords bilatéraux avec l'UE devraient être un pas vers une amélioration et une extension des relations mutuelles. J'expose brièvement ci-après pourquoi il me parait important que le peuple puisse se prononcer sur des changements de ce genre, lourds de conséquences. L'accord sur le transport terrestre sera source d'énormes problèmes avec l'afflux de poids lourds qu'il amènera et les charges financières qui en découleront. A l'avenir, la Suisse devra dépenser quelque 300 millions de francs par année au titre de mesures d'appoint pour le transfert de la route au rail. En outre, le flux des 40 tonnes causera de graves dégradations aux ponts et chaussées qui entraîneront à leur tour des frais considérables, sans parler des nuisances. Il est illusoire de croire que le trafic pourra passer de la route au rail dans la mesure espérée. L'introduction de la libre concurrence dans le domaine de l'agriculture profitera certes aux consommateurs en provoquant une baisse des prix des produits alimentaires mais engendrera aussi des problèmes et des coûts dans le secteur agricole au détriment des agriculteurs. En définitive, seule la grande industrie bénéficiera des accords bilatéraux. Les PME, les classes moyennes et les indépendants en seront pour leurs frais.

Pour leur part, les médecins sont directement concernés par la libre circulation des personnes. Croire qu'après sept ans un frein pourra y être mis par la voie d'un référendum facultatif n'est qu'illusion. Un rejet entraînerait en effet l'annulation des six autres accords. A partir de 2001, sera instituée la reconnais- sance mutuelle des diplômes. Cela signifie que le niveau très élevé de formation du corps médical suisse va s'abaisser et que nous aurons un nivellement par le bas. De plus, durant les prochaines années, il faudra compter avec une augmentation annuelle d'environ 380 médecins suisses en pratique privée. Dès 2001 également, il faudra y ajouter entre 30 et 215 ouvertures de cabinets médicaux par des médecins étrangers travaillant déjà en Suisse. Passé 2007, les «eurodocs" seront également autorisés à ouvrir un cabinet en Suisse. Il est cependant permis de penser que leur nombre sera limité en raison d'un niveau de formation inférieur à celui exigé par la FMH. Les coûts du système de santé engendrés par la croissance médicale augmenteront au rythme d'environ 265 millions de francs par année, soit 1 milliard tous les quatre ans. Jusqu'ici, le nombre total des médecins en pratique privée augmentait de 300 à 400 par année, à l'avenir la croissance annuelle sera de 500 à 600. L'attrait d'une nouvelle existence en Suisse est considérable, d'autant plus que le réseau social de notre pays offre de nombreux avantages. La libre circulation des personnes conduira à une surpopulation universitaire. Actuellement, on dénombre déjà quelque 15000 étudiants de l'UE en Suisse. Le numerus clausus paraît inévitable, car l'agrandissement des universités entraînerait de nouvelles charges financières.

Le paquet des sept accords bilatéraux est contraire aux intérêts de la Suisse. De plus, seul un non clair lors de la votation du 21 mai 2000 empêchera une adhésion à l'UE pour les prochaines années. 